\title{
Le récit de voyage polonais : entre anthropologie et reportage
}

\author{
Anne-Marie Monluçon \\ Université de Grenoble Alpes/UMR 5316 Litt \& Arts
}

En Europe occidentale, les voyages lointains ont, trop souvent, été associés aux conquêtes et à la mise en place d'empires coloniaux, qui ont impliqué savants, militaires, missionnaires, marchands et parfois artistes. En va-t-il autrement pour les grands voyageurs ou reporters polonais ? Leur pays n'a pas eu, a priori, d'empire colonial, si bien que le reporter Ryszard Kapuściński se présentait parfois, en Afrique, comme un blanc doté d'une expérience, ou du moins d'un passé de colonisé, en référence aux partages de la Pologne ${ }^{1}$. Certains objecteront que les Kresy, les territoires des confins, aux périodes où la Pologne s'étendait sur une partie des Pays baltes, de la Biélorussie et de l'Ukraine, invitent à nuancer le propos. Mais de colonies lointaines, point. Les Polonais se sont-ils pour autant désintéressés des contrées éloignées ? Le colloque organisé du 21 au 23 octobre 2015, à l'Université Grenoble Alpes, consacré à «La Pologne citoyenne du monde : regards croisés sur les écrivains voyageurs polonais $\left(\mathrm{XIX}^{\mathrm{e}}-\mathrm{XXI}^{\mathrm{e}}\right.$ siècles) ${ }^{2} \gg$ a démontré le contraire.

1. À partir de 1772, la Prusse, l'Autriche et la Russie s'emparent progressivement du territoire polonais pour le faire disparaître de la carte des États européens en 1795. Cette situation perdurera jusqu'en 1918.

2. Ce colloque a pu se tenir grâce au soutien du ministère de l'Enseignement supérieur et de la Recherche, de l'Université Stendhal-Grenoble 3, de l'E.A Traverses 19-21, de l'E.A. Ilcea4, de la Fondation Jan Michalski, du Consulat général de Pologne de Lyon, de Grenoble-Alpes Métropole, de l'Institut Polonais et de l'Instytut Książki [Institut du livre] de Cracovie. La première partie des articles nourris par le colloque a été publiée dans MonluÇon \& SAIGNES, 2016. 
SLOVO

10 Les Voyages lointains des écrivains polonais $\left(\mathrm{XX}^{\mathrm{e}}-\mathrm{XXI}^{\mathrm{e}}\right.$ siècles $)-\mathrm{n}^{\circ} 51$

Chaque génération de Polonais est parvenue à expédier vers des destinations lointaines quelques artistes, savants ou reporters, quel que soit le contexte politique (partages de la Pologne, entre 1772 et 1918 ; enfermement et censure durant la période de la République populaire de Pologne). On n'est pas surpris de trouver, parmi les auteurs étudiés dans le présent volume, une majorité de contemporains, souvent nés sous le régime socialiste, et ayant commencé leur carrière littéraire ou journalistique autour de 1989. Cette date ouvre une période de démocratisation du voyage et du tourisme, dans les pays de l'ancien bloc de l'Est, même si les destinations lointaines ne sont accessibles qu'à une minorité de privilégiés. Elle ouvre aussi une période d'essor de la presse, enfin libre, qui devient - notamment grâce au quotidien Gazeta Wyborcza [Le Journal électoral], crée en 1989 - une véritable pépinière pour quelques-unes des plus belles plumes du reportage littéraire polonais : Jacek Hugo-Bader, Andrzej Stasiuk, Wojciech Tochman, Mariusz Wilk...

Un rapide parcours de notre table des matières révèle des manques et un déséquilibre qui méritent un commentaire. Un ouvrage collectif résulte toujours d'une part de contingence et d'une recherche de cohérence. On ne trouvera pas dans ce volume d'article consacré à Joseph Conrad ni à Ryszard Kapuściński. L'un et l'autre ont été l'objet de nombreux travaux, plus rarement en français qu'en polonais dans le cas de Kapusciński ${ }^{3}$, il est vrai. Ces auteurs sont toutefois présents indirectement presque à chaque page de ce volume, en tant que pères fondateurs, modèles auxquels se réfèrent nombre de leurs héritiers contemporains étudiés ici, tantôt sur le mode de l'hommage, tantôt de manière plus critique, tel Mariusz Wilk qui dénonce la « méthode de Kapuściński ».

Ce dernier exemple illustre bien le correctif qu'impose le contenu du volume en regard de ce que pourraient laisser croire les intitulés des rubriques et les titres des articles. La première d'entre elles, « De la Russie au Japon » paraît disproportionnée, avec six articles, dont quatre traitent essentiellement de la Russie, ou plus exactement de ses marges : le Grand Nord où a longtemps résidé Mariusz Wilk et l'Extrême-Orient russe, visité par Ferdynand Ossendowski et Jacek Hugo-Bader. La surreprésentation de la Russie, tsariste ou post-soviétique peut s'expliquer de diverses manières. Tout d'abord, les Polonais ont souvent eu à subir l'hégémonie politique de leur grand voisin, apprendre sa langue, voire considérer la Sibérie comme une « seconde patrie » ainsi que le note Ferdynand Ossendowski dans sa préface d'Asie fantôme. Lors de ses missions de géologue, il y a rencontré des Polonais descendants de déportés du bagne tsariste ${ }^{4}$. Les récits de déportation,

\section{Monluçon, 2012.}

4. Le regard de F. Ossendowski sur la Sibérie gagnerait à être confronté aux travaux des 
par exemple ceux de Józef Czapski et de Gustaw Herling-Grudziński, constituent d'ailleurs des monuments dans la littérature polonaise $\mathrm{du} \mathrm{xx}^{\mathrm{e}}$ siècle, mais ces déplacements forcés ne sont pas l'objet de ce volume car ils ne peuvent être considérés de la même manière que les voyages désirés et choisis. Les violences de l'Histoire expliquent cependant en partie la curiosité et la familiarité des écrivains et reporters polonais à l'égard de la Sibérie et des autres lointaines régions d'Asie centrale. En second lieu, l'impossibilité de la dissociation au sein de cette rubrique, qui englobe les voyages vers des destinations situées entre l'est de la Russie et le

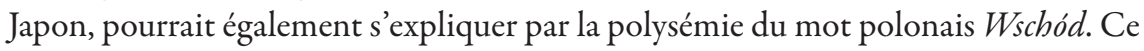
n'est pas là uniquement une question d'extension territoriale de la Russie, puissance eurasiatique, ni même de l'empan des pérégrinations de nos auteurs. En effet, le terme Wschód désigne en polonais plusieurs choses : l'Est, c'est-à-dire un point cardinal ; l'Orient, donc un tropisme et un imaginaire ; et enfin le Levant, le lieu où se lève l'astre solaire (du verbe polonais wschodzić [se lever]). Andrzej Stasiuk

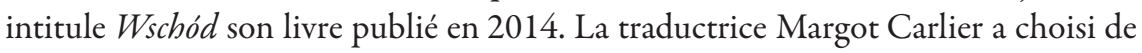
traduire par L'Est. Pourtant, le livre contient, à côté de souvenirs d'enfance et de récits de retours de l'auteur dans l'Est de la Pologne (région natale de ses parents), des récits de grands voyages en Chine et en Mongolie. Toutes ces destinations se résument en un seul mot : wschód, impossible à traduire en français avec la richesse de ses nuances. Enfin, la rubrique consacrée aux voyages « De la Russie au Japon » semble suffisamment étoffée pour ébaucher une approche diachronique de la représentation de ces contrées de 1898 à 2014 (malgré l'absence, dans ce volume, d'articles écrits à l'époque de la République populaire de Pologne). La Russie soviétique est, bien sûr, très présente dans les récits de ces voyages vers «l'Est » : dans les ouvrages d'Ossendowski, d'Hugo-Bader et de Wilk, ainsi que chez Stasiuk, dont le regard rétrospectif embrasse à la fois l'expérience soviétique, mongole et chinoise, tout en y mêlant les propres souvenirs d'enfance de l'auteur dans l'est de la Pologne populaire. Tous ces livres s'inscrivent, d'une manière ou d'une autre, dans la continuité d'Imperium, l'ouvrage de Kapusciński publié en 1993, qui regroupe les récits des voyages du reporter en Union soviétique puis en Russie, composant ainsi une chronique fragmentaire de la lente décomposition du système.

La seconde rubrique consacrée au pôle opposé, l'Amérique du Nord, l'Occident de l'Occident, paraît, en comparaison avec la précédente, de taille réduite, avec seulement deux articles. Notons tout d'abord qu'aucun des deux auteurs abordés n'a été traduit en français. Ce volume en propose donc, au lecteur francophone, une première approche. Chaque texte correspond à une époque

spécialistes de littérature française réunis dans Moussa \& STROEv, 2014. 
SLOVO

12 Les Voyages lointains des écrivains polonais $\left(\mathrm{XX}^{\mathrm{e}}-\mathrm{XXI}^{\mathrm{e}}\right.$ siècles $)-\mathrm{n}^{\circ} 51$

différente. Arkady Fiedler voyage au Canada dans les années 1930 et publie le récit de son voyage, Kanada pachnaca żywica [Le Canada qui sent bon la sève] en 1935. Miron Białoszewski, pour sa part, séjourne au États-Unis en octobre et novembre 1982. Aleksander Fiut analyse son récit de voyage intitulé AAmeryka [AAmérique], publié à titre posthume, en 1988 et son Tajny dziennik [Journal secret], paru seulement en 2012.

Notons cependant que l'Amérique est également présente en filigrane dans les textes consacrés aux voyages vers l'Est: dans Bêtes, hommes et dieux et Asie fantôme de Ferdynand Ossendowski, comme le démontrent les articles d'Elżbieta Koziołkiewicz et d'Anne-Marie Monluçon. En effet, lorsqu'Ossendowski parvient à quitter l'Asie, en 1921, il se réfugie d'abord aux États-Unis, où il conçoit Bêtes, hommes et dieux, en 1922, sous la pression de son éditeur et de son traducteur/ co-auteur qui l'encouragent à adopter les critères esthétiques du roman d'aventures et du western anglo-saxons, lesquels se retrouvent aussi dans Asie fantôme, écrit en Pologne l'année suivante. Tout un système d'analogies géographiques et historiques entre Far West et Far East sous-tend également ses ouvres. L'ouvrage de Fiedler, étudié par Piotr Bilos, revient sur la formation de ce pays constitué par une mosaique de populations dont la représentation porte l'empreinte des modèles romantiques. Mais il serait erroné de croire que Fiedler est le premier voyageur polonais à écrire sur le Nouveau Monde. Il faut resituer ce texte dans une tradition de voyages et reportages qui commencent au XVIII ${ }^{e}$ siècle, avec les mémoires d'outre-Atlantique de Tomasz Kajetan Węgierski et qui se poursuit au XIX ${ }^{\mathrm{e}}$ avec, parmi d'autres, Listy $z$ Ameryki [Lettres d'Amérique] de Henryk Sienkiewicz, et la trilogie de Wańkowicz intitulée $W$ ślady Kolumba [Sur les traces de Christophe Colomb] publiée entre 1967 et 1969.

La troisième rubrique intitulée «Suds» propose un raccourci saisissant. L'expédition australe de Bronisław Malinowski et de Stanisław Ignacy Witkiewicz, dans les années 1914-1918, est sans doute l'un des derniers exemples de l'association traditionnelle du savant et de l'artiste. On peut dire qu'elle marque les débuts d'une forme de modernité : à l'issue de ce voyage, en 1922, Bronisław Malinowski a, en écrivant Les Argonautes du Pacifique occidental, donné à l'anthropologie sa charte fondatrice; Witkiewicz, lui, après avoir passé en Russie les années 1914-1918, a exploré les limites de l'art figuratif, en peinture et au théâtre, et entrepris de réinventer le roman. L'autre texte étudié est le reportage au long cours de Wojciech Tochman, escorté d'un guide interprète qui est en même temps un survivant et un témoin du génocide rwandais. Ce texte, qui raconte comment des hommes se sont mis un jour à tuer massivement leurs voisins, relève de l'ère du témoin.

Le lecteur ne manquera pas de remarquer qu'un seul article étudie l'œuvre d'une auteure, en l'occurrence l'article de Marzena Karwowska consacré à Japoński wachlarz [L'Éventail japonais] de Joanna Bator (2004), anthropologue 
de formation ayant travaillé sur le féminisme. En réalité, même si les voyages lointains sont longtemps restés une affaire d'hommes, le féminin est présent à plusieurs niveaux du corpus étudié dans ce volume. Ferdynand Ossendowski rend un hommage appuyé à sa mère, qui lui a donné le goût de la nature sauvage, tandis que l'article de Katia Vandenborre consacré à Hugo-Bader ébauche une filiation entre l'auteur et la lauréate du Prix Nobel de littérature en 2015. Si les reporters polonais contemporains sont tous des fils de R. Kapusciński, ils ont deux mères : Svetlana Alexievitch et Hanna Krall.

La présence du féminin introduit-elle le thème d'Éros en voyage ? Le désir occupe une place discrète dans la galerie des personnages féminins que représente Ferdynand Ossendowski dans Asie fantôme, avec deux pôles assez contrastés et sans doute stéréotypés: les très jeunes filles tartares, opprimées et séduisantes, et les héroïnes tragiques russes des histoires enchâssées. Les premières relèvent sans doute d'un certain orientalisme et les secondes, assurément, de modèles romanesques. Joanna Bator suggère avec discrétion que la métamorphose qu'opère sur elle son séjour au Japon, ou plus exactement le regard que l'on y porte sur son physique, a sans doute une incidence sur son érotisme. Parmi les rares textes de notre corpus où Éros affirme sa présence, les œuvres parallèles de Witkiewicz et de Malinowski sont l'objet d'un renouvellement critique de la part de Stanisław Jasionowicz et d'Anna Saignes. Le tiraillement entre les désirs érotiques les moins censurés et le renoncement ne procède pas d'un puritanisme religieux catholique polonais ou britannique victorien mais, d'une part, de la tension pour parvenir à faire œuvre et, d'autre part, de l'expérience consciente d'une «non identité du sujet à soi », problématique éminemment moderne. Mais c'est Miron Białoszewski qui aborde la sexualité de la façon la plus explicite, dans son Journal secret, publié bien après sa mort.

La problématique principale de ce volume reste cependant la suivante : le voyage lointain est-il toujours corrélé avec l'expérience de l'Altérité ? S'agit-il uniquement de l'Altérité culturelle, ou peut-elle être sociale ? Cette Altérité est-elle vécue avec plaisir ou déplaisir ? Peut-elle échapper à l'exotisme et à ses clichés ? Quelles formes et quel sens peut prendre le dépassement de l'Altérité ? La découverte d'universaux qui font de l'Autre un autre nous-mêmes ? Au fil du temps, observe-t-on une érosion de l'Altérité, une « fin du voyage » (comme le pensait Claude Lévi-Strauss), due à l'uniformisation et à la mondialisation ? À quelle connaissance de soi mène l'expérience de l'Autre ? À une découverte de notre identité culturelle, c'est-à-dire de notre appartenance à un groupe ou bien plutôt à une identité individuelle, voire à l'Autre qui est en nous, pour parler comme Rimbaud ?

Dans les voyages lointains et les récits qu'on peut en rapporter, on peut distinguer trois niveaux d'altérité : le premier concerne la langue comme instrument de communication ou, à l'inverse, obstacle et matière de la création littéraire; le 
SLOVO

14 Les Voyages lointains des écrivains polonais $\left(\mathrm{XX}^{\mathrm{e}}-\mathrm{XXI}^{\mathrm{e}}\right.$ siècles $)-\mathrm{n}^{\circ} 51$

deuxième est lié à l'expérience sur le terrain comme expérience de la familiarité ou, au contraire, de l'étrangeté que nous inspire l'Autre, ou encore, paradoxalement, comme retrouvailles avec le Même; le troisième tient à la culture, bibliothèque littéraire ou théorique, réserve de genres et de formes qu'emporte l'écrivain voyageur dans ses valises et qui informe sa perception, son expérience et son écriture.

\section{Altérité linguistique et circulation des textes}

Le corpus ici étudié propose un large éventail de cas de figure concernant la langue, comprise et parlée, en voyage. Certains auteurs sont de savants polyglottes comme Ossendowski, maniant entre autres le russe, le mongol et le chinois. Wilk mâtine le polonais de mots et expressions russes. Malinowski déjà avait parsemé son Journal, rédigé en polonais, de mots et expression anglais. Mais les difficultés liées à la langue apparaissent de plus en plus clairement dans le récit. Hugo-Bader affirme qu'il s'est spécialisé dans l'espace russophone parce qu'il maîtrise bien cette langue et considère que c'est une condition nécessaire du reportage, rappelle Katia Vandeborre. Jacek Milewski, que Piotr Bilos confronte à Arkady Fiedler, a appris le romani pour l'enseigner aux Tziganes polonais de la région de Suwałki, dans le Nord-Est du pays. Parmi les contemporains, tous utilisent peu ou prou l'anglais, comme Wojciech Tochman au Rwanda avec son jeune guide et interprète, Léonard. Pour Joanna Bator, bien que celle-ci s' initie au japonais, cette langue tierce constitue un espace de liberté et de transgression bilatérales, ainsi que le montre Marzena Karwowska. Une certaine tradition du récit de voyage a longtemps accrédité une sorte de féérie linguistique, en omettant de préciser dans quelle langue ont eu lieu, pendant le voyage, les échanges, qui étaient généralement rapportés au style indirect libre, par souci d'élégance et de lissage narratif. Le voyageur y apparaissait comme un polyglotte pour qui les obstacles linguistiques n'existaient pas. Le genre du reportage a contribué à faire des conditions d'accès à l'information un véritable sujet.

Sur le plan de l'écriture, on peut repérer deux types de situations opposées qui donnent, chacune, à penser. Les auteurs qui peuvent écrire et penser dans leur langue maternelle insistent souvent sur le retour à leurs propres langue et à la littérature, nécessaire pour reconstituer leur expérience, y compris celle de l'Altérité. Stanisław Jasionowicz et Anna Saignes mettent en évidence l'enracinement de Malinowski dans le contexte littéraire et artistique polonais de la fin du XIX siècle, qui éclaire dans une mesure certaine l'expérience de l'Altérité dont rend compte le Journal d'ethnographe. Isabelle Després accomplit le même travail sur la dimension réflexive du Journal d'un loup de Mariusz Wilk. Cet écrivain avait, lors d'une rencontre avec le public grenoblois, en octobre 2015, insisté sur le rôle des 
dictionnaires et de la profondeur historique de sa langue, puisant dans le polonais du XVII ${ }^{e}$ siècle et le slavon. Les lecteurs du recueil Autoportrait d'un reporter ${ }^{5}$ savent que R. Kapusciński se déplaçait toujours avec certains classiques polonais et des dictionnaires. L'enracinement dans sa propre langue et sa littérature pour dire l'Altérité est donc l'un des paradoxes rencontrés à la lecture de ces auteurs.

À l'opposé, nombre d'auteurs polonais voués à l'exil, par choix ou sous la pression éditoriale, ont écrit en anglais. Ces textes ont souvent suivi un cheminement sinueux pour parvenir jusqu'à nous. Le best-seller d'Ossendowski est traduit en anglais, à partir d'un récit oral d'Ossendowski, en 1922, avant d'être rédigé en polonais. Malinowski, tout comme son modèle Conrad, écrit en anglais son livre fondateur de l'anthropologie, Les Argonautes du Pacifique occidental (2002), mais pendant son séjour sur l'île Mailu et les îles Trobriand, il rédige un journal en polonais, qui sera publié d'abord en anglais en 1967, puis traduit en français à partir de l'anglais. Il ne sera publié en polonais qu'en 2002. Les études consacrées ici à Ossendowski et Malinowski soulignent combien les éditeurs et traducteurs ont pris de libertés avec les textes, qu'ils n'ont pas hésité à couper, redécouper, adapter, américaniser, lisser. La perspective diachronique pointe alors un progrès. Les auteurs polonais contemporains bénéficient aujourd'hui d'une traduction directe. Les lecteurs francophones doivent au travail de Véronique Patte de pouvoir lire enfin l'œuvre de Kapusciński, traduit directement depuis le polonais ${ }^{6}$. Gardons-nous cependant de trop d'optimisme. Beaucoup de textes, récents ou anciens, n'ont toujours pas été traduits, grand nombre d'ouvrages sont aujourd'hui épuisés, et on continue à publier à l'identique des traductions anciennes de textes polonais via l'anglais, généralement contestables. L'obstacle de la langue ne concerne pas seulement la circulation des hommes, mais aussi celle des textes.

\section{Sur le terrain : expérience de l'Altérité et rapport à soi}

Sur le terrain, les ouvres dont traitent ici les chercheurs représentent, elles aussi, une typologie variée du rapport à l'Autre et à Soi. Stasiuk, adepte des incursions brèves et répétées, dans le Sud-Est de l'Europe, est tout de même obligé d'allonger ses séjours quand il s'agit d'arpenter les steppes d'Asie. Hugo-Bader et Tochman pratiquent le «grand reportage » long et lointain. Malinowski prend ses quartiers pour plusieurs mois sur l'île Mailu puis les îles Trobriand. Wilk illustre le cas particulier du résident étranger, établi sine die, tel un Durrell en Grèce, jadis.

5. KAPUSCINSKI, 2008, p. 53 et 82.

6. KAPUSCINSKI, 2014. 
SLOVO

16

Les Voyages lointains des écrivains polonais $\left(\mathrm{XX}^{\mathrm{e}}-\mathrm{XXI}^{\mathrm{e}}\right.$ siècles $)-\mathrm{n}^{\circ} 51$

Le regard porté sur l'Autre ne trahit pas seulement l'ouverture ou, au contraire, l'étroitesse d'esprit du voyageur. Il a aussi partie liée avec ce que la censure de l'époque permet, ou ne permet pas, d'écrire. Plusieurs auteurs écrivent un « deuxième livre », pour reprendre l'expression de Vincent Debaene ${ }^{7}$, désignant un phénomène qu'il étudie dans le domaine français. Il en va ainsi d'Ossendowski, qui rédige rapports scientifiques et récits de voyage aventureux. Deux d'entre eux (Malinowski et Białoszewski) écrivent en parallèle du livre officiel un journal secret qui sera publié à titre posthume, ce qui nous rappelle le poids du contexte politique mais aussi moral et religieux qui pèse sur le récit de voyage. Ces deux œuvres gémellaires rendent compte d'une ambivalence face à l'Altérité, vécue tantôt comme souffrance, tantôt comme plaisir.

Le rapport au même ou à soi est un autre fil que l'on peut tirer de l'écheveau des textes. On est surpris par le nombre de compatriotes expatriés que côtoient voyageurs et reporters polonais et le regard très variable que ceux-ci portent sur ceux-là : héros romantiques pour Fiedler, modèles éducatifs pour la jeunesse chez Ossendowski et moines Pallotins sévèrement critiqués par Tochman, qui reconstitue le rôle de l'Église polonaise lors du génocide rwandais. Le voyage lointain peut modifier le rapport à notre groupe d'origine et à nous-mêmes. Ainsi Marzena Karwowska retrace-t-elle la dynamique de L'Éventail japonais comme une conversion intellectuelle de Joanna Bator, d'abord convaincue par la thèse de R. Barthes, dans L'Empire des signes, selon lequel on peut découpler langue et connaissance, s'affranchir du langage verbal, elle rallie les thèses opposées de Wittgenstein et Cassirer : la langue nous donne accès à la connaissance tout en la découpant et en la structurant. Par exemple, le japonais est une langue profondément hiérarchique et genrée, et étroitement liée en cela aux structures sociales japonaises. L'expérience japonaise l'amène à remettre totalement en cause sa position théorique initiale.

Les cas de refus de l'Altérité, ou du moins de refus avoué, sont rares parmi les voyageurs et reporters, a priori curieux et ouverts. Malinowski jette les bases de l'anthropologie du $\mathrm{Xx}^{\mathrm{e}}$ siècle qui intègre «l'ethnologie participante », attitude de rapprochement actif vis-à-vis de l'Autre, que pratique spontanément Ossendowski ou son contemporain, le Russe Vladimir Arseniev, l'auteur de Dersou Ouzala. Mais la gamme des trajectoires reste nuancée pour Ossendowski : il est difficile de trancher si l'Altération de soi, refusée in extremis, est jouée ou sincère. À l'autre extrémité, Wilk, en Russie, se défait de sa polonité et devient « un des leurs », donc Autre, jusqu'à modifier la langue dans laquelle il écrit. On retrouve cependant assurément un clivage entre les différencialistes comme Fiedler et Milewski et 
les universalistes, tel Malinowski, qui perçoit dans les sociétés trobriandaises les linéaments de la liberté et de la démocratie chères aux Européens. Les œuvres présentent également un nuancier qui va de l'orientalisme au constat de la « fin du voyage » pour reprendre, une fois de plus, la formule de Claude Lévi-Strauss. À l'heure de la mondialisation, on retrouve partout les mêmes objets manufacturés, les mêmes écrans. Les louanges de l'Altérité peuvent sonner comme l'expression d'un désir égoïste de préserver l'exotisme à tout prix, quitte à souhaiter le blocage des sociétés traditionnelles et à refuser, sans les consulter, leur évolution. La déploration de l'uniformisation, de l'érosion de l'Altérité, est-elle fondée sur un constat lucide ou est-elle héritière de l'Orientalisme ? En tout cas, la « fin du voyage » ne coïncide pas avec la fin des récits de voyage ou des reportages, le second relayant en partie le premier, sur le plan de l'Histoire littéraire et de l'évolution des genres.

\section{Altérités intellectuelles : intertexte théorique, générique et formel}

Contrairement à ce qu'on pourrait croire, le bagage intellectuel et esthétique des voyageurs et reporters ne provient pas uniquement de leur culture nationale. Leur regard est informé par diverses sources qui trahissent des transferts culturels, ainsi qu'une hybridation générique et formelle en amont du voyage et bien sûr en aval, durant le temps de l'écriture. L'écriture viatique polonaise se situe, comme toute autre, au carrefour de la littérature et des sciences humaines, et à la croisée d'une tradition nationale et internationale. L'anthropologie est bien représentée dans ce volume, par la figure tutélaire de Malinowski mais aussi par celle, beaucoup plus récente, de Bator qui a abandonné une carrière de chercheur en anthropologie en 2011 pour se consacrer à l'écriture. Et l'on sait tout ce que Kapusciński doit aux sciences humaines (historiographie, anthropologie...) dont il se réclame dans des livres réflexifs sur son métier, comme Mes voyages avec Hérodote, Autoportrait d'un reporter ${ }^{8}$ ou les volumes de Lapidarium (non traduits en français).

La bibliographie des articles de ce recueil révèle que la riche bibliothèque théorique et littéraire des auteurs inclut de nombreux auteurs germanophones (Ernst Mach, Ernst Cassirer, Max Scheler, après Nietzsche et Schopenhauer), ou anglophones (Marshall McLuhan, Clifford Geertz, James Clifford) ainsi que français (Gabriel Marcel, Jean Baudrillard, Emmanuel Lévinas, Roland Barthes, Tzvetan Todorov). Les sources polonaises signalent, à l'inverse, ce que les lecteurs français peuvent déjà lire comme les ouvrages du sociologue Zygmunt Bauman ou la monographie de Beata Nowacka et Zygmunt Ziątek, Ryszard Kapuściński. 
SLOVO

18 Les Voyages lointains des écrivains polonais $\left(\mathrm{XX}^{\mathrm{e}}-\mathrm{XXI}^{\mathrm{e}}\right.$ siècles $)-\mathrm{n}^{\circ} 51$

Biographie d'un écrivain ${ }^{9}$. Les délais de traduction, et, encore une fois, la médiation récurrente de l'anglais sont révélatrices des processus de transferts culturels. Les idées ne se propagent pas toutes au rythme de leur production. Leur diffusion rencontre, comme celle des œuvres, des obstacles sauf quand les chercheurs polyglottes lisent en version originale ou en traduction anglaise. Et une fois de plus, l'on espère voir combler, un jour, grâce à des éditeurs et des traducteurs de bonne volonté, certaines lacunes. Ainsi les lecteurs polonais connaissent-ils le Japon de Roland Barthes, et, depuis 2014, celui de Nicolas Bouvier, dans ses Chroniques japonaises ${ }^{10}$.

L'un des acquis de ce volume concerne assurément les perspectives de comparatisme littéraire qu'ouvrent les repérages d'intertextualité par les chercheurs : récits d'aventures anglo-saxons, allemands ou polonais chez Ossendowski, appropriation intime de la littérature russe, en particulier chez Wilk, dialogue serré avec l'œuvre de Witkiewicz autant qu'avec celle de Conrad chez Malinowski (et réciproquement). Le volume fait aussi apparaître la nécessité de situer les auteurs polonais par rapport à un certain nombre d'œuvres incontournables, telles la trilogie d'Arseniev, le célèbre travelogue de Jack Kerouac, les monuments de la littérature des camps (Soljénitsyne, Chalamov, Herling-Grudziński), la trilogie de Jean Hatzfeld, les œuvres sur la fin de l'homme rouge de Svietlana Alexievitch.

Sur le plan générique, les voyages lointains ainsi que le grand reportage témoignent de la même diversité que les voyages de proximité présentés dans le volume de Recherches \& Travaux consacré aux « Voyages en Europe des écrivains polonais »: la dimension autobiographique est inhérente au récit de voyage et présente chez Malinowski, Białoszewski, Stasiuk, Bator..., contrairement au reportage où la subjectivité du journaliste ne s'exprime pas sous la forme d'une narration autobiographique mais par l'implication dans le choix des sujets, des témoins et par la qualité de l'écoute, du commentaire. Avec des modalités différentes, Hugo-Bader et Tochman proposent des récits de voix et cultivent le voisinage entre reportage et témoignage. L'essayisme est moins présent bien qu'il soit l'une des marques de fabrique de Stasiuk par exemple, comme le fait ressortir l'analyse d'Agnieszka Kaczmarek consacrée au temps et à la mélancolie chez cet auteur. Les récits de voyages ou reportages littéraires mêlent à la fois littérature du réel et subjectivité assumée. Ainsi quand un Wańkowicz revendiquait le droit à la fiction, il posait avant tout la question suivante : quels sont les critères d'une fiction acceptable? Cette question se pose aujourd'hui avec encore plus d'acuité, car, par étape, le climat intellectuel a changé. Tout d'abord, à l'Ouest, depuis les

9. NowACKa \& ZiąTEK, 2019.

10. BOUVIER, 2014. 
années 1985, le négationnisme et d'autres formes de révisionnisme ont soudain dramatisé les enjeux. La situation actuelle, avec le problème des fake news et de ce que l'on appelle la post-vérité, renforce cette évolution. Ces questions, d'une importance capitale, ne doivent pas nous faire perdre de vue que le voyage est toujours l'occasion de confronter le mythe à la réalité et que le récit de voyage et certains reportages littéraires conjuguent traditionnellement littérature du réel et puissance de l'imaginaire, sur une ligne de crête où la création tente de l'emporter sur les stéréotypes, forme de l'imaginaire collectif figé, qui est le plus sûr moyen de manquer la réalité.

Sur le plan poétique, les voies de la création se déploient entre deux pôles : celui d'une écriture très libre, « en suivant le pinceau » pour Joanna Bator, en renouant avec le genre des « Notes» pour Wilk, voire du silva rerum ${ }^{11}$, ou celui d'une écriture à contrainte, telle que Hugo-Bader la pratique dans Fièure blanche, avec des chapitres-tableaux à la manière de Kapusciński, restituant le rythme du voyage en autostop.

La part de l'imaginaire et de la fiction affaiblit-elle le potentiel d'intervention dans le monde du récit de voyage et du reportage, pourtant réputé pour ses rapports avec l'engagement ? Ces deux genres ont suscité des vocations de lecteurs voyageurs, y compris dans des milieux moins favorisés que les traditionnels globe-trotters aristocrates. Deux auteurs contemporains, Hugo-Bader et Stasiuk sont des autodidactes qui élargissent la sociologie des rencontres. Historiquement, la littérature viatique, quand elle n'a pas figé et essentialisé l'Autre, a contribué à l'humaniser et à décoloniser le regard des Occidentaux, en montrant, entre autres, les lointaines conséquences des faits et gestes de nos pays.

Mais la surprise vient d'un autre côté. Le départ au loin, et surtout loin des économies dites «développées», est souvent un voyage dans le temps, vers l'enfance, le passé. Or nous rencontrons ici la mélancolie, le sentiment d'une perte qui ne se limite pas au passé, qui n'est pas simple nostalgie, car elle mine la jouissance du présent et notre capacité à nous projeter dans l'avenir. Peut-être faudrait-il distinguer entre la mélancolie des textes du début $\mathrm{du} \mathrm{Xx}^{\mathrm{e}}$ siècle et ceux de nos contemporains. La perte de la métaphysique, chez Witkiewicz, coïncide, historiquement, avec le « désenchantement du monde » théorisé par le sociologue Max Weber en 1917, pour désigner le processus de recul des croyances religieuses et magiques au profit des explications scientifiques. Andrzej Stasiuk, écrivain de l'Invisible s'il en est, en serait alors le lointain héritier. Mais le thème $\mathrm{du}$ «paradis » $[\mathrm{raj}]$ terrestre, menacé ou disparu, chez Ossendowski et Fiedler, 
SLOVO

20 Les Voyages lointains des écrivains polonais $\left(\mathrm{XX}^{\mathrm{e}}-\mathrm{XXI}^{\mathrm{e}}\right.$ siècles $)-\mathrm{n}^{\circ} 51$

est plus complexe à interpréter. La réception que nous en faisons aujourd'hui lui confère des résonnances avec nos préoccupations les plus contemporaines : signes précurseurs de l'effondrement des écosystèmes et de la biodiversité ou prémices d'une conscience écologique ? Il faut se garder de nos projections anachroniques : en premier lieu, les deux auteurs sont très ambivalents à l'égard de la Nature et du Progrès technique, et l'on sait bien que l'écologie n'a cessé de changer de camp, qu'il en existe dès l'origine des versions réactionnaires et d'autres progressistes; en second lieu, il ne faut pas sous-estimer l'influence des schèmes romantiques ou déclinistes de l'époque. Il n'empêche que leurs livres nous amènent à relire ces temps que nous percevions comme ceux du commencement, de l'aube du $\mathrm{XX}^{\mathrm{e}}$ siècle, comme le commencement de notre possible fin. Chez nos contemporains, Wilk, Hugo-Bader et Stasiuk, la mélancolie procède sans doute autant d'une stratification de processus historiques que de la sensibilité ou de la posture individuelle. Pour cette génération devenue adulte dans les années 1980, la « fin des grands récits » ou des « hautes espérances », pour faire écho au film de Mike Leigh, en 1988 - foi en le Progrès, la Révolution ou le Socialisme - était acquise, et sans doute plus libératrice que mélancolique. Mais leurs textes font un bilan des dégâts sociaux, écologiques et culturels hérités de la période soviétique ainsi que de ceux qui se développent dans les sociétés post-soviétiques. Ils prennent sans doute acte du désenchantement politique de l'après 1989 et se font l'écho de la dévastation, irréversible en maints endroits, du patrimoine naturel et culturel. Parmi ceux-ci, dès 2014, dans L'Est, Andrzej Stasiuk, déploie une stratégie originale pour s'adresser à sa mère, auditrice de Radio Maria et aux électeurs qui s'apprêtent à porter au pouvoir le parti Droit et Justice en octobre 2015, car il combine la plus grande empathie avec la critique la plus frontale, ce que lui permet son parcours de «transfuge social par le haut », pour reprendre la terminologie de Pierre Bourdieu. Il faudrait, là encore, faire la part du lointain héritage romantique, si prégnant dans la culture polonaise, et d'une intuition remarquable des situations. Mais ne faudrait-il pas creuser aussi du côté de l'Imaginaire - rêverie sur les origines ou projets et utopies passés - du fait de la récurrence frappante du mot «paradis » $[\mathrm{raj}]$ dans les titres de livres et de chapitres des auteurs polonais (chapitre intitulé « Le Paradis du chasseur » dans Asie fantôme d'Ossendowski, chapitre « Il n'y aura pas de paradis » dans La Guerre $d u$ foot et autres guerres de Kapuściński ${ }^{12}, W$ rajskiej dolinie, wśród zielska [Dans la vallée paradisiaque, parmi les mauvaises herbes] d'Hugo-Bader ${ }^{13}$, À chacun son

12. KAPUSCINSKI, 1978.

13. Hugo-BADER, 2010. 
paradis de Mariusz Szczygiet ${ }^{14}$ ) ? Déclinés avec des significations et des tonalités variées, ils nous interdisent de n'y voir qu'une référence biblique, car il s'agit, dans certains cas, du paradis terrestre perdu.

Faire circuler les textes n'est donc pas tout à fait vain et produit non seulement quelques rencontres avec le réel mais parfois des effets sur le réel. En témoigne la très récente émergence d'une réception des écrivains voyageurs polonais parmi les écrivains français. L'un des premiers, François Bon a salué Sur la route de Babadag de Stasiuk sur son $\operatorname{blog}^{15}$; dans L'Hiver aux trousses. Voyages en Russie d'Extrême-Orient ${ }^{16}$, Cédric Gras marche en partie sur les traces d'Arseniev et Ossendowski qu'il mentionne explicitement, tandis qu'Emmanuel Ruben, sous le titre Sur la route du Danube ${ }^{17}$, rend explicitement hommage à Sur la route de Babadag de Stasiuk, même si son maître reste Nicolas Bouvier. Ces deux derniers, nés dans les années 1980, sont géographes de formation comme Arseniev. Les transferts culturels sont certes toujours inachevés et dissymétriques mais désormais en cours de rééquilibrage, afin que les écrivains polonais, et plus largement ceux de l'Est, nous paraissent un peu moins Autres, un peu plus familiers.

Le manque étant l'une des figures du désir, concluons par la liste des chantiers qu'il reste à ouvrir: que les écrivains polonais soient enfin publiés en France avec leurs photos ou leurs illustrations, dont ils sont systématiquement privés, à l'exception de Fièure blanche éditée chez Noir sur Blanc, de manière à faciliter les études inter-médiales qui s'imposent, que nos travaux contribuent aussi à orienter les traductions du français vers le polonais, devenues rares et répondant trop souvent à des critères commerciaux.

\section{Bibliographie sélective (la plupart des références sont données à la fin des différents articles du volume)}

\section{OEures}

BARTHEs Roland, 1970, L’Empire dessignes, éd. Skira/Flammarion (coll. Champs), Paris, 151 p. [Trad. en polonais par Dziadek Adam, 1999, Imperium znaków, Wydawnictwo KR, Varsovie, 200 p.]

14. SZCZYGIEe, 2010.

15. Bon, 10. 09.2007.

16. GRAS, 2015.

17. RubEN, 2019. 
SLOVO

22 Les Voyages lointains des écrivains polonais $\left(\mathrm{Xx}^{\mathrm{e}}-\mathrm{XXI}^{\mathrm{e}}\right.$ siècles $)-\mathrm{n}^{0} 51$

Bouvier Nicolas, 2004 (1989), Chronique japonaise in CEuvres, Quarto Gallimard, Paris, 1428 p. [En polonais : Kronika japońska. Pustka i petnia, przeł. Krystyna Sławińska, Krystyna Arustowicz, Oficyna Literacka Noir sur Blanc, Warszawa 2014, 350 p.]

Gras Cédric, 2015, L'Hiver aux trousses: voyage en Russie d'Extrême-Orient, Stock, Paris, 272 p.

Hugo-BADER Jacek, 2010, W rajskiej dolinie wśród zielska [Dans la vallée paradisiaque, parmi les mauvaises herbes], Wydawnictwo Czarne, Wołowiec, 400 p.

Kapuscinski Ryszard, 1978, Wojna futbolowa, Czytelnik, Varsovie, 242 p. [En français, 2004, Il n'y aura pas de paradis. La Guerre du foot et autres guerres et aventures, trad. du polonais par PATTE Véronique, Pocket, Paris, 320 p.]

KAPUSCINSKi Ryszard, 2008 (2003), Autoportrait d'un reporter, trad. du polonais par Patte Véronique, Plon (coll. Feux croisés), Paris, 180 p.

Kapuscinski Ryszard, 2014, OEuvres, trad. du polonais par Patte Véronique, Flammarion, Paris, 1488 p. [Ce volume réunit les nombreuses œuvres du reporter, traduites directement du polonais, sans constituer pour autant des CEuvres complètes.]

Ruben Emmanuel, 2019, Sur la route du Danube, Payot \& Rivages, Paris, 608 p.

SzCzygię Mariusz, 2010, Zrób sobie raj, Wołowiec, Wydawnictwo Czarne, 292 p. [En français, 2012, Chacun son paradis, trad. du polonais par CARLIER M., Éditions Actes Sud, Arles, 267 p.]

\section{Critiques}

Debaene Vincent, 2010, L'Adieu au voyage, Gallimard (coll. Bibliothèque des sciences humaines), Paris, $528 \mathrm{p}$.

Monluçon Anne-Marie \& Saignes Anna (dir.), 2016, Les Voyages en Europe des écrivains polonais (XIX-XXI siècles) in Recherches \& Travaux, $\mathrm{n}^{\circ} 89,170 \mathrm{p}$. https://journals.openedition.org/recherchestravaux/830. 
Monluçon Anne-Marie, 2012, «Ryszard Kapuściński et Andrzej Stasiuk, deux écrivains polonais à fronts renversés ? » in La Chute de l'URSS. Une fin d'empire. Recherches \& Travaux, $\mathrm{n}^{\circ}$ 80, p. 141-171, http://journals.openedition. org/recherchestravaux/526.

Moussa Sarga \& Stroev Alexandre, 2014, L'Invention de la Sibérie par les voyageurs et écrivains français (XVIII-XIX ${ }^{e}$ siècles), Institut d'études slaves, Paris, $234 \mathrm{p}$.

Nowacka Beata \& Ziątek Zygmunt, 2019, Ryszard Kapuściński. Biographie d'un écrivain, Peter Lang, Warszawa, $416 \mathrm{p}$.

\section{Sitographie}

Bon François, 10.09.2007, https://www.tierslivre.net/spip/spip.php?article975 (consulté le 06/07/2019). 